\title{
CD4+:CD8+ T Cell Ratio Normalization and the Development of AIDS Events in People with HIV Starting Antiretroviral Therapy
}

\author{
Hajra Okhai, ${ }^{1}$ María Jesús Vivancos-Gallego, ${ }^{2}$ Teresa Hill, ${ }^{1}$ and Caroline A. Sabin ${ }^{1}$
}

\begin{abstract}
We identify factors associated with the normalization of the CD4+:CD8+ $\mathrm{T}$ cell ratio among UK Collaborative HIV Cohort study participants, and describe the association of the CD4+ and CD8+ T cell counts and the CD4+:CD8+ T cell ratio, with the risk of new AIDS events among individuals who achieve a suppressed viral load. Participants initiating combination antiretroviral therapy (cART) after 2006 with a CD4+:CD8+ T cell ratio $<1$, and viral suppression within 6 months were included. Cox proportional hazard models were used to examine associations with ratio normalization (ratio $\geq 1$ ). Poisson regression models were used to investigate factors associated with the development of AIDS after viral load suppression. A total of 13,178 participants [median age: 37 (interquartile range: $31-44)$ ] were followed for 75,336 person-years. Of the 4,042 (32.9\%) who experienced ratio normalization, individuals with a high $\mathrm{CD} 4+\mathrm{T}$ cell count $\left[>500 \mathrm{vs} . \leq 200 \mathrm{cells} / \mathrm{mm}^{3}\right.$, adjusted hazard ratio (95\% confidence interval): 7.93 (6.97-9.01)], low CD8+ T cell count $\left[>1,150\right.$ vs. $\leq 500$ cells $/ \mathrm{mm}^{3}: 0.18(0.16-$ $0.21)$ ], and low CD4+:CD8+ T cell ratio [ $>0.8$ vs. $<0.2: 12.36(10.41-14.68)]$ at cART initiation were more likely to experience ratio normalization. Four hundred and nineteen people developed a new AIDS event. Most recent CD4+ T cell count $\left[>500\right.$ vs. $\leq 200$ cells $/ \mathrm{mm}^{3}$ : adjusted rate ratio $\left.0.24(0.16-0.34)\right]$ and CD4+:CD8+ T cell ratio [ $>0.8$ vs. $<0.2: 0.33(0.21-0.52)]$ were independently associated with a new AIDS event. One third of study participants experienced ratio normalization after starting cART. CD4+ T cell count and CD4+:CD8+ T cell ratio are both individually associated with ratio normalization and the development of new AIDS events after cART.
\end{abstract}

Keywords: CD4+ T cells, CD4+:CD8+ T cell ratio, antiretroviral therapy, HIV

\section{Introduction}

$\mathbf{U}$ NTREATED HIV INFECTION is characterized by a derangement of the immune system, with reductions in the CD4+ T cell count, increases in the CD8+ T cell count, and an abnormally low CD4+:CD8+ T cell ratio, ${ }^{1}$ as well as increased levels of inflammatory markers, with changes occurring soon after seroconversion. ${ }^{2}$ Treatment with combination antiretroviral therapy (cART) generally results in rapid suppression of HIV viremia and improvements in immune markers-however, in some individuals, immune markers remain abnormal, despite viral suppression. In particular, even among those who experience improvements in their CD4+ and
$\mathrm{CD} 8+\mathrm{T}$ cell counts, the $\mathrm{CD} 4+\mathrm{CD} 8+\mathrm{T}$ cell ratio may remain lower than would be expected in people without HIV. ${ }^{3}$

In adults with HIV with virological suppression, the CD4+:CD8+ T cell ratio inversely correlates with measures of innate and adaptive immune senescence. ${ }^{4}$ We have previously reported that the pretreatment CD4+ T cell count is strongly associated with CD4+:CD8+ T cell ratio recovery, ${ }^{5}$ but it is still unclear if prolonged treatment with antiretroviral therapy (ART) will ultimately result in a full restoration of the immune system. Further research into the dynamics of the CD4+:CD8+ T cell ratio may help to understand the contribution of these measures to immune restoration post-ART.

\footnotetext{
${ }^{1}$ Centre for Clinical Research, Epidemiology, Modelling and Evaluation, Institute for Global Health, University College London, London United Kingdom.

${ }^{2}$ Department of Infectious Diseases, University Hospital Ramon y Cajal and Ramon y Cajal Health Research Institute (IRYCIS), Madrid, Spain.

(C) Hajra Okhai, et al., 2020; Published by Mary Ann Liebert, Inc. This Open Access article is distributed under the terms of the Creative Commons License (http://creativecommons.org/licenses/by/4.0), which permits unrestricted use, distribution, and reproduction in any medium, provided the original work is properly cited.
} 
In this study, we aim to identify factors associated with the normalization of the CD4+:CD8+ $\mathrm{T}$ cell ratio among participants in the UK Collaborative HIV Cohort (UK CHIC) study, and to describe the association of CD4+ and $\mathrm{CD} 8+\mathrm{T}$ cell counts, as well as the CD4+:CD8+ T cell ratio, with the risk of new AIDS events among individuals who achieve a suppressed viral load.

\section{Methods}

\section{Study participants}

The UK CHIC study is an ongoing cohort of HIV-positive individuals ( $>16$ years of age), who have accessed care at one or more of $25 \mathrm{HIV}$ clinics in the United Kingdom at any time from 1996 onward. The study methods are described elsewhere. ${ }^{6}$ In brief, centers collect data on demographic information, ART treatment history, laboratory results, and AIDS diagnoses; the resulting dataset is submitted on an annual basis to the coordinating center. The project was approved by a Multicenter Research Ethics Committee (MREC/00/7/47) and by local ethics committees.

The analyses described here are based on data collected up to December 31, 2017. Participants were included in the analyses if their CD4+:CD8+ $\mathrm{T}$ cell ratio was $<1$ pretreatment initiation, and they were antiretroviral naive, started cART after 2006, were followed in the study for at least 6 months after starting cART, and experienced HIV viral load suppression during this period. In addition, participants were required to have at least one CD4+ and $\mathrm{CD} 8+\mathrm{T}$ cell count measurement within the 6-month periods both before and after starting cART. Sensitivity analyses expanding the time period for suppression to 8 months were done to ensure our study did not favor individuals with more rapid suppression of viral load.

\section{Statistical analysis}

Longitudinal CD4+:CD8+ $\mathrm{T}$ cell ratios were calculated using raw $\mathrm{CD} 4+$ and $\mathrm{CD} 8+\mathrm{T}$ cell counts where both values were reported on the same date. Continuous variables were expressed as the median and interquartile range (IQR), and categorical variables as counts and percentages. To explore CD4+:CD8+ $\mathrm{T}$ cell ratio dynamics, the closest recorded ratios at $6,12,18$, and 24 months after cART initiation were selected. Summary estimates were calculated for each time point with no restriction on availability of CD4+:CD8+ T cell ratio and were stratified by $\mathrm{CD} 4+\mathrm{T}$ cell count and $\mathrm{CD} 4+\mathrm{CD} 8+$ $\mathrm{T}$ cell ratio at treatment initiation.

Ratio normalization was defined as a value $>1$ based on previously published studies. ${ }^{7}$ Individuals with a normalized ratio within 6 months of cART initiation were excluded from analyses of factors associated with normalization - this was to ensure that the inclusion of individuals with a pre-cART ratio that was close to 1 , in whom only minor fluctuations to the CD4+ and CD8+ T cell counts may result in a normalized ratio, did not reduce our ability to detect clinically important changes in the ratio.

For analyses of the time to ratio normalization, follow-up started on the date of ART initiation and was right censored on the earliest of date of ratio normalization, date of death, date of loss to follow-up, or December 31, 2017. Cox proportional hazard $(\mathrm{PH})$ regression models were used to examine associations of demographic and clinical variables with this outcome. In particular, we examined associations with ethnic origin (white, black, or other), mode of HIV acquisition (sex between men, sex between men and women, or other/unknown), gender, age at treatment initiation, CD4+ and $\mathrm{CD} 8+\mathrm{T}$ cell count/CD4+:CD8+ T cell ratio/HIV viral load at cART initiation, hepatitis $B$ virus (HBV)/hepatitis $\mathrm{C}$ virus (HCV) status, AIDS status at treatment initiation, initial treatment regimen [regimens, including either nonnucleoside reverse transcriptase inhibitors (NNRTIs), protease inhibitors (PIs), integrase strand transfer inhibitors (INSTIs), or other combinations], regimen backbone [abacavir/lamivudine (ABC/TTC), zidovudine/lamivudine (ZDV/TTC), or tenofovir disoproxil fumarate/emtricitabine (TDF/FTC)], and calendar period of treatment initiation. As the ratio is calculated using the CD4+ $\mathrm{T}$ cell and $\mathrm{CD} 8+\mathrm{T}$ cell counts, multivariable models were built separately for $\mathrm{CD} 4+$ and $\mathrm{CD} 8+\mathrm{T}$ cell counts and CD4+:CD8+ T cell ratio. Variables significantly associated $(p<.05)$ with ratio normalization in univariate analyses were selected for multivariable models.

We used Poisson regression models to investigate factors associated with the development of AIDS after cART. Individuals with an AIDS diagnosis before cART initiation were excluded from this analysis as we were particularly interested in the development of new AIDS events only. Follow-up for these analyses started at 6 months after ART initiation, so as to ensure time for the person to have achieved viral suppression and to exclude any AIDS event that may have been unmasked or caused by immune restoration inflammatory syndrome, and ended at the earliest of the date of first recorded AIDS event, date of death, date of loss to follow-up, or December 31, 2017. We examined associations with demographic factors, mode of HIV acquisition, and clinical data both at cART initiation and as time-updated covariates (CD4+ and CD8+ T cell counts, as well as the ratio, $\mathrm{HBV} / \mathrm{HCV}$ status); although all participants were required to have experienced viral suppression in the first 6 months after cART initiation, we did not censor follow-up at virological rebound-instead, analyses also included adjustment for the latest HIV viral load as a time-updated covariate. In a similar manner to the models of ratio normalization, multivariable models were developed separately to include either the $\mathrm{CD} 4+$ and $\mathrm{CD} 8+\mathrm{T}$ cell counts or the CD4+:CD8+ T cell ratio.

\section{Results}

\section{Participant characteristics}

A total of 27,785 UK CHIC participants were identified to have started ART between 2006 and 2017. Individuals were excluded if they had a normalized CD4+:CD8+ T cell ratio in the 6 months before initiating cART $(n=1,149)$, had $<6$ months follow-up $(n=1,998)$, did not achieve viral suppression within 6 months of initiating cART $(n=6,626)$, or did not have immunological data pre-ART and post-ART initiation $(n=4,834)$. The remaining 13,178 UK CHIC participants were followed for a total of 75,336 person-years.

The majority of participants were male (78.8\%), of white ethnicity $(59.1 \%)$, and acquired HIV through sex with men (61.2\%) (Table 1). At the time of ART initiation, participants had a median age of 37 (IQR: 31-44) years had a median CD4+ $\mathrm{T}$ cell count of 311 (IQR: 208-440) cells $/ \mathrm{mm}^{3}$ and a median $\mathrm{CD} 4+: \mathrm{CD} 8+\mathrm{T}$ cell ratio of 0.3 (IQR: 0.2-0.5). Most participants initiated a cART regimen that included a NNRTI (59.4\%). However, since the rollout 
Table 1. Characteristics of the UK Collaborative HIV Cohort Participants Included in the Study

n (\%) or median (IQR) as appropriate
Total

Age at UK CHIC entry

(median, IQR), years

Sex, $n(\%)$

Male

Female

Ethnicity, $n(\%)$

White

Black

Other/unknown

Mode of HIV acquisition, $n(\%)$

Sex between men

Sex between men and women

Other/unknown

Year of cART initiation

2009-2014

2015-2017

initiation (copies/mL)

$<10,000$

$10,000-100,000$

100,001-500,000

$>500,000$

Unknown

CD4+ T cell count at cART

initiation (median, IQR), cells $/ \mathrm{mL}$

CD8+ $\mathrm{T}$ cell count at $\mathrm{cART}$ initiation (median, IQR), cells $/ \mathrm{mL}$

CD4+:CD8+ $\mathrm{T}$ cell ratio at cART initiation (median, IQR), cells/mL

cART regimen, $n(\%)$

NNRTI

PI

INSTI

Other
2006-2008

HIV viral load at cART

13,178 (100.0)

$37(31-44)$

$10,390(78.8)$

2,787 (21.2)

7,791 (59.1)

$3,561(27.0)$

$1,826(13.9)$

$8,063(61.2)$

4,139 (31.4)

$976(7.4)$

3,384 (25.7)

$7,534(57.2)$

2,260 (17.1)

4,906 (37.2)

4,851 (36.8)

2,478 (18.8)

$525(4.0)$

418 (3.2)

$311.0(208.0-440.0)$

$940.0(670.0-1,313.0)$

$0.3(0.2-0.5)$

$7,825(59.4)$

$3,385(25.7)$

$1,473(11.2)$

495 (3.8)

Regimen backbone at cART initiation, $n(\%)$ TDF/FTC

ZDV/TTC

$9,970(75.7)$

$\mathrm{ABC} / \mathrm{TTC}$

$502(3.8)$

$2,301(17.5)$

Other

$405(3.1)$

AIDS event before cART initiation, $n(\%)$

No

$11,441(86.8)$

Yes

$1,737(13.2)$

HBV at cART initiation, $n(\%)$

No/unknown

Yes

$12,873(97.7)$

$305(2.3)$

$\mathrm{HCV}$ at cART initiation, $n(\%)$

No/unknown

Yes

$12,578(95.4)$

$600(4.6)$

$\%$, percentage; ABC/TTC, abacavir and lamivudine; cART, combination antiretroviral therapy; $\mathrm{HBV}$, hepatitis $\mathrm{B}$ virus; $\mathrm{HCV}$, hepatitis $B$ virus; INSTI, integrase strand transfer inhibitors; IQR, interquartile range; $n$, number of participants; NNRTI, non-nucleoside reverse transcriptase inhibitors; PI, protease inhibitors; TDF/FTC, tenofovir disoproxil fumarate and emtricitabine; UK CHIC, UK Collaborative HIV Cohort; ZDV/TTC, zidovudine and lamivudine. of INSTIs from 2015, these have become increasingly common as part of first-line cART regimens $(11.2 \%$ of those initiating cART overall, but $48.6 \%$ of those initiating cART between 2015 and 2017).

\section{Rate of normalization}

Figure 1 shows the median $\mathrm{CD} 4+: \mathrm{CD} 8+\mathrm{T}$ cell ratio 6 monthly after starting cART, stratified by the absolute CD4+ T cell count (Fig. 1A) and the CD4+:CD8+ T cell ratio (Fig. 1B) at baseline. Over 24 months, the median CD4+:CD8+ T cell ratio doubled from 0.3 at month 0 to 0.6 at month 24. Increases were similar regardless of the absolute CD4+ $\mathrm{T}$ cell count or $\mathrm{CD} 4+\mathrm{CD} 8+\mathrm{T}$ cell ratio at cART initiation (0.24-0.35).

Of the 13,178 participants, 946 were excluded from further analysis as their CD4+:CD8+ T cell ratio had already normalized within the first 6 months of cART initiation. Of the remaining 12,232 participants, 4,042 (32.9\%) achieved normalization over a median of 2.3 years.

Results from univariable and multivariable Cox PHs models are presented in Table 2. In univariable analyses, participants who initiated treatment in more recent years with a higher CD4+ $\mathrm{T}$ cell count or higher CD4+:CD8+ T cell ratio, or who initiated an INSTI-based regimen (vs. NNRTI) were more likely to experience a normalization of their ratio. In contrast, older individuals, those acquiring HIV through heterosexual exposure (vs. sex between men), those of black ethnicity (vs. white), those with an AIDS diagnosis at treatment initiation, those with HBV, and those initiating regimens, including PIs (vs. NNRTI), $\mathrm{ABC} / \mathrm{TTC}$, or ZDV/TTC (vs. TDF/FTC), were all less likely to experience normalization of their ratio. No associations were seen with sex, HCV, or HIV viral load at cART initiation.

Similar results were seen in the multivariable models (Table 2), with those who were older at cART initiation, those of a black, other/unknown ethnic group (vs. white), and those who initiated a PI-based regimen (vs. NNRTI) or one including either ABC/TTC or ZDV/TTC (vs. TDF/FTC) being less likely to experience normalization of their ratio. In addition, individuals who had AIDS, HCV infection, or a high CD8+ T cell count at treatment initiation were also less likely to experience normalization of their ratio. Participants with higher $\mathrm{CD} 4+\mathrm{T}$ cell counts at treatment initiation were more likely to experience ratio normalization. In contrast to univariable models, individuals acquiring HIV through heterosexual contact had a higher likelihood of experiencing ratio normalization, after adjusting for confounders. Sensitivity analyses removing participants with a baseline CD4+:CD8+ $\mathrm{T}$ cell ratio of $>0.8$ showed similar results.

\section{Risk of a new AIDS event according to the latest CD4+} and $C D 8+T$ cell counts and the $C D 4+: C D 8+T$ cell ratio

A total of 1,737 participants who already had an AIDS event before cART initiation were excluded from these analyses. Among the remaining 11,442 people followed for 60,440 person-years, 419 people developed at least one new AIDS event after cART initiation. The majority of the participants with a new AIDS event were men $(81.6 \%, 342 / 419)$ who had sex with men $(64.4 \%, 270 / 419)$ and of white ethnicity $(61.3 \%$, 257/419). A large proportion of the AIDS events were unspecified (21.2\%, 89/419); however, of those that were, herpes simplex disease was the most reported AIDS event $(52.4 \%$, 
FIG. 1. Median (IQR) CD4+:CD8+ $\mathrm{T}$ cell ratio at cART initiation, $6,12,18$, and 24 months after cART initiation $(n=13,178)$, stratified by (A) CD4+ T cell count and (B) CD4+:CD8+ $\mathrm{T}$ cell ratio at cART initiation. Line reference at $\mathrm{CD} 4+\mathrm{CD} 8+\mathrm{T}$ cell ratio $=1 . \mathrm{cART}$, combination antiretroviral treatment; $\mathrm{IQR}$, interquartile range.
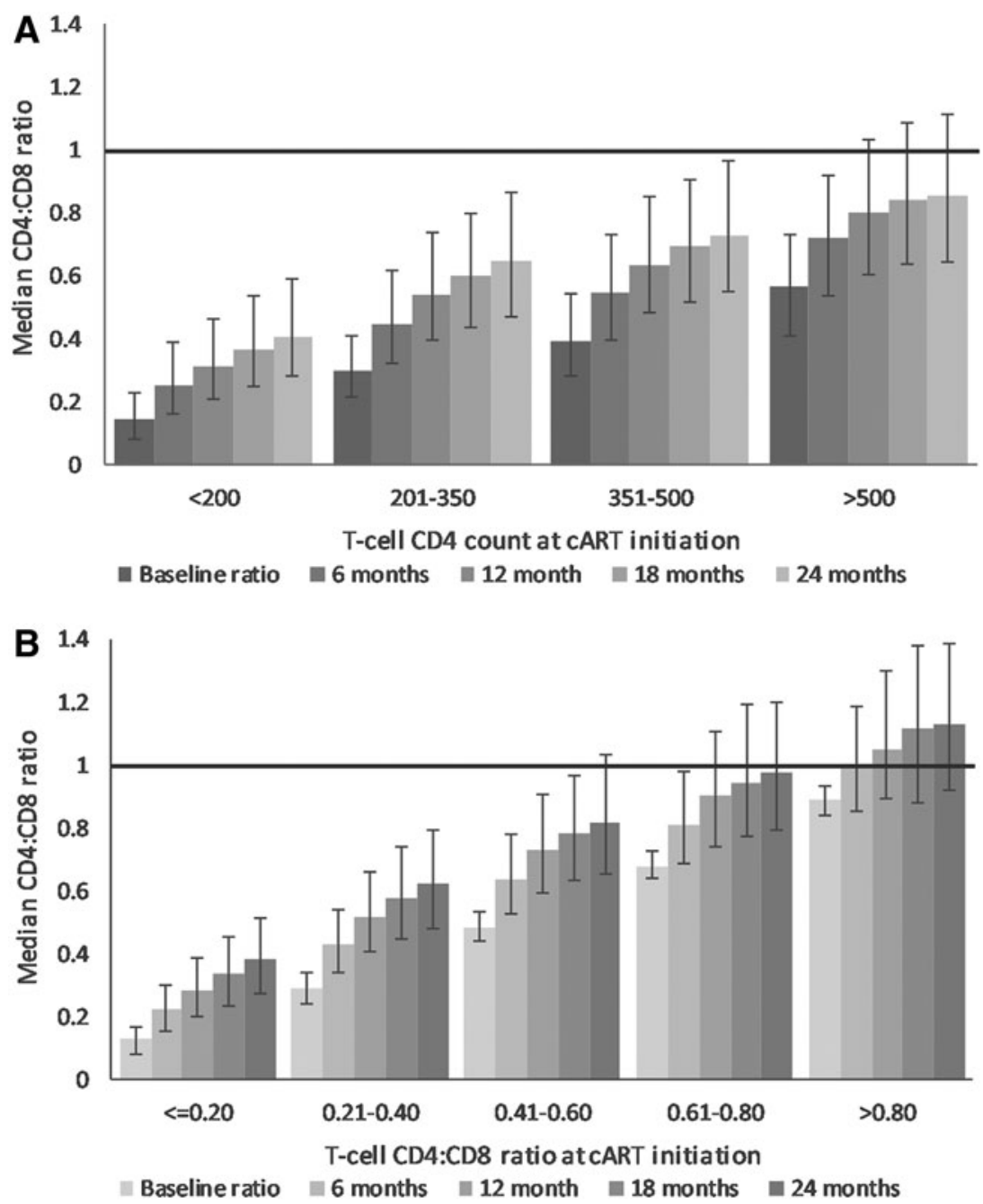

173/330), followed by Mycobacterium tuberculosis complex (14.6\%, 48/330) and Kaposi's sarcoma (7.3\%, 24/330).

Univariable analyses (not shown) suggested that older age, sex, ethnicity, mode of acquisition of HIV, HCV infection, HIV viral load, CD4+ T cell count, and CD4:CD8+ T cell ratio were associated with the development of a new AIDS event. Multivariable models (Table 3) suggested that, while there was no association between the CD4+ T cell count or CD4:CD8+ T cell ratio at the time of ART initiation and the development of a new AIDS event, both measures were associated with a new AIDS event when assessed over follow-up in a time-updated manner. No association was seen between the CD8+ T cell count and the development of a new AIDS event, whether considered a fixed (at ART initiation) or time-updated (over time) covariate. Analyses were repeated, expanding the period of viral suppression to 8 months, with similar conclusions.

\section{Discussion}

In this observational cohort study, we found that only around a third of UK CHIC participants attaining viral suppression on cART achieved normalization of their CD4+:CD8+ $\mathrm{T}$ cell ratio over a median follow-up time of
6 years. A higher CD4+ T cell count, lower CD8+ T cell count, and higher $\mathrm{CD} 4+: \mathrm{CD} 8+\mathrm{T}$ cell ratio at the time of initiating cART were each associated with an increased chance of achieving ratio normalization. Of those who had attained viral suppression within the first 6 months after starting cART, only $3.7 \%$ went on to develop a new clinical AIDS event. The latest CD4+ T cell count and CD4+:CD8+ T cell ratio were both individually strongly associated with the development of a new AIDS events on cART.

The proportion of individuals achieving ratio normalization in our study is similar to that reported by Tinago et al. ${ }^{8}$ in Ireland (26.3\%) and Mussini et al. ${ }^{7}$ in Italy $(29.4 \%)$, but is higher than that reported in a more recent study in Thailand, ${ }^{9}$ where only $18.6 \%$ of participants experienced normalization of their CD4+:CD8+ ratio over 5 years of follow-up. Of note, in the general population, an inverted CD4+:CD8+ $\mathrm{T}$ cell ratio is uncommon with only $8 \%$ of people $20-59$ years of age in a Swedish cohort having a CD4+:CD8+ T cell ratio $<1,{ }^{10}$ although the prevalence of an inverted ratio did increase with age. One possible explanation for the higher proportion with a ratio $<1$ in our study compared to the Thai study may be earlier diagnosis ${ }^{4}$ and treatment initiation at higher CD4+ T cell counts; the pretreatment CD4+ T cell count in the Thai cohort was only 206 cells $/ \mathrm{mm}^{3}$ compared to 311 cells $/ \mathrm{mm}^{3}$ in 
Table 2. Factors Associated with Time to CD4+:CD8+ T Cell Ratio Normalization (Ratio >1), Univariable and Multivariable Cox Proportional Hazard Models (Models Were Developed Separately for the CD4+ and CD8+ T Cell Counts Themselves and the CD4+:CD8+ T Cell Ratio)

\begin{tabular}{|c|c|c|c|c|c|c|}
\hline \multirow[b]{2}{*}{ Variable } & \multicolumn{2}{|c|}{ Univariable models } & \multicolumn{4}{|c|}{ Multivariable models } \\
\hline & $H R(95 \% C I)$ & $\mathrm{p}$ & $\begin{array}{c}\text { (i) with absolute CD4+ } \\
\text { and CD8+ T cell counts } \\
\text { aHR }(95 \% \text { CI })\end{array}$ & $\mathrm{p}$ & $\begin{array}{c}\text { (ii) with CD4+:CD8+ } \\
T \text { cell ratio } \\
\text { aHR }(95 \% \text { CI })\end{array}$ & $\mathrm{p}$ \\
\hline \multicolumn{7}{|c|}{ CD4+ $\mathrm{T}$ cell count at cART initiation $\left(\right.$ cells $\left./ \mathrm{mm}^{3}\right)$} \\
\hline$\leq 200$ & 1 & .0001 & 1 & .0001 & & \\
\hline $201-350$ & $2.03(1.85-2.23)$ & & $3.05(2.75-3.38)$ & & & \\
\hline $351-550$ & $2.42(2.19-2.68)$ & & $4.61(4.10-5.18)$ & & & \\
\hline$>500$ & $3.45(3.11-3.84)$ & & $7.93(6.97-9.01)$ & & & \\
\hline \multicolumn{7}{|c|}{ CD8+ T cell count at cART initiation $\left(\right.$ cells $\left./ \mathrm{mm}^{3}\right)$} \\
\hline$\leq 500$ & 1 & .0001 & 1 & .0001 & & \\
\hline $501-750$ & $1.22(1.10-1.36)$ & & $0.65(0.58-0.73)$ & & & \\
\hline $751-1,150$ & $0.93(0.84-1.02)$ & & $0.38(0.34-0.42)$ & & & \\
\hline$>1,150$ & $0.56(0.51-0.63)$ & & $0.18(0.16-0.21)$ & & & \\
\hline \multicolumn{7}{|c|}{$\mathrm{CD} 4+: \mathrm{CD} 8+\mathrm{T}$ cell ratio at $\mathrm{cART}$ initiation } \\
\hline$\leq 0.20$ & 1 & .0001 & & & 1 & .0001 \\
\hline $0.21-0.40$ & $2.52(2.27-2.78)$ & & & & $2.52(2.27-2.79)$ & \\
\hline $0.41-0.60$ & $5.47(4.93-6.08)$ & & & & $5.69(5.11-6.34)$ & \\
\hline $0.61-0.80$ & $7.92(7.01-8.96)$ & & & & $8.53(7.52-9.68)$ & \\
\hline$>0.80$ & $11.01(9.31-13.02)$ & & & & $12.36(10.41-14.68)$ & \\
\hline $\begin{array}{l}\text { Age at cART initiation/ } \\
10 \text { years older }\end{array}$ & $0.89(0.86-0.92)$ & .0001 & $0.92(0.89-0.96)$ & .0001 & $0.93(0.90-0.97)$ & .0001 \\
\hline \multicolumn{7}{|l|}{ Sex } \\
\hline Male & 1 & .08 & & & & \\
\hline Female & $1.07(0.99-1.15)$ & & & & & \\
\hline \multicolumn{7}{|l|}{ Ethnicity } \\
\hline White & 1 & .0001 & 1 & .0001 & 1 & .003 \\
\hline Black & $0.77(0.72-0.83)$ & & $0.81(0.73-0.90)$ & & $0.85(0.76-0.94)$ & \\
\hline Other/unknown & $0.92(0.83-1.01)$ & & $0.88(0.80-0.97)$ & & $0.91(0.83-1.01)$ & \\
\hline \multicolumn{7}{|l|}{ Mode of HIV acquisition } \\
\hline Sex between men & 1 & .0001 & 1 & .0001 & 1 & .0001 \\
\hline $\begin{array}{l}\text { Sex between men } \\
\text { and women }\end{array}$ & $0.85(0.79-0.91)$ & & $1.23(1.11-1.35)$ & & $1.25(1.13-1.37)$ & \\
\hline Other/unknown & $0.89(0.78-1.01)$ & & $0.93(0.82-1.06)$ & & $0.91(0.80-1.03)$ & \\
\hline \multicolumn{7}{|c|}{ HIV viral load at cART initiation } \\
\hline $\log _{10}$ copies $/ \mathrm{mL}$ & $1.01(0.98-1.03)$ & .65 & & & & \\
\hline \multicolumn{7}{|c|}{ AIDS event before cART } \\
\hline No & 1 & .0001 & 1 & .0006 & 1 & .007 \\
\hline Yes & $0.70(0.63-0.77)$ & & $0.84(0.76-0.93)$ & & $0.87(0.79-0.96)$ & \\
\hline \multicolumn{7}{|l|}{$\mathrm{HBV}$ at cART initiation } \\
\hline No & 1 & .002 & 1 & .005 & 1 & .02 \\
\hline Yes & $0.72(0.58-0.90)$ & & $0.73(0.58-0.91)$ & & $0.77(0.61-0.96)$ & \\
\hline \multicolumn{7}{|l|}{$\mathrm{HCV}$ at $\mathrm{cART}$ initiation } \\
\hline No & 1 & .71 & & & & \\
\hline Yes & $0.97(0.84-1.13)$ & & & & & \\
\hline \multicolumn{7}{|l|}{ Initial cART regimen } \\
\hline NNRTI & 1 & .0001 & 1 & .0001 & 1 & .002 \\
\hline PI & $0.86(0.80-0.92)$ & & $0.85(0.79-0.92)$ & & $0.86(0.80-0.93)$ & \\
\hline INSTI & $1.19(1.05-1.34)$ & & $1.01(0.88-1.17)$ & & $1.00(0.87-1.14)$ & \\
\hline Other & $0.92(0.77-1.10)$ & & $0.92(0.74-1.14)$ & & $0.98(0.79-1.21)$ & \\
\hline \multicolumn{7}{|l|}{ Regimen backbone } \\
\hline TDF/FTC & 1 & .0002 & 1 & .0003 & 1 & .0001 \\
\hline ABC/TTC & $0.90(0.82-0.97)$ & & $0.89(0.82-0.97)$ & & $0.86(0.79-0.94)$ & \\
\hline ZDV/TTC & $0.75(0.64-0.89)$ & & $0.55(0.46-0.65)$ & & $0.49(0.41-0.58)$ & \\
\hline Other & $0.84(0.70-1.02)$ & & $0.78(0.62-0.98)$ & & $0.69(0.55-0.86)$ & \\
\hline \multicolumn{7}{|l|}{ Year of cART initiation } \\
\hline 2006-2008 & 1 & .0001 & 1 & .001 & 1 & .0001 \\
\hline 2009-2014 & $1.11(1.04-1.19)$ & & $0.87(0.81-0.94)$ & & $0.83(0.77-0.89)$ & \\
\hline $2015-2017$ & $1.44(1.28-1.61)$ & & $0.92(0.80-1.05)$ & & $0.83(0.72-0.95)$ & \\
\hline
\end{tabular}

95\% CIs, 95\% confidence intervals; aHR, adjusted hazard ratio; HR: hazard ratio. 
Table 3. Relative Rate of Aids Events; Results Shown Are from Four Multivariable Poisson Models

\begin{tabular}{|c|c|c|c|c|}
\hline \multirow[b]{2}{*}{ Variable } & \multicolumn{2}{|c|}{ A. Covariates at treatment initiation } & \multicolumn{2}{|c|}{ B. Latest covariates over follow-up } \\
\hline & $R R(95 \% C I)$ & $\mathrm{p}$ & $R R(95 \% C I)$ & $\mathrm{p}$ \\
\hline \multicolumn{5}{|c|}{ Model 1} \\
\hline \multicolumn{5}{|c|}{ CD4+ T cell count $\left(\right.$ cells $\left./ \mathrm{mm}^{3}\right)$} \\
\hline$\leq 200$ & 1 & .14 & 1 & .0001 \\
\hline $201-350$ & $0.79(0.61-1.02)$ & & $0.38(0.25-0.57)$ & \\
\hline $351-500$ & $0.71(0.52-0.98)$ & & $0.33(0.23-0.49)$ & \\
\hline$>500$ & $0.89(0.62-1.27)$ & & $0.24(0.16-0.34)$ & \\
\hline \multicolumn{5}{|c|}{ CD8+ T cell count (cells $\left./ \mathrm{mm}^{3}\right)$} \\
\hline$\leq 500$ & 1 & .37 & 1 & .16 \\
\hline $501-750$ & $0.93(0.65-1.32)$ & & $1.25(0.89-1.76)$ & \\
\hline $751-1,150$ & $0.83(0.60-1.17)$ & & $1.34(0.96-1.87)$ & \\
\hline$>1,150$ & $1.02(0.73-1.44)$ & & $1.49(1.04-2.12)$ & \\
\hline \multicolumn{5}{|c|}{ Model 2} \\
\hline \multicolumn{5}{|c|}{ CD4+:CD8+ $\mathrm{T}$ cell ratio } \\
\hline$\leq 0.20$ & 1 & .09 & 1 & .0001 \\
\hline $0.21-0.40$ & $0.79(0.62-1.00)$ & & $0.60(0.38-0.95)$ & \\
\hline $0.41-0.60$ & $0.66(0.49-0.90)$ & & $0.51(0.33-0.79)$ & \\
\hline $0.61-0.80$ & $0.87(0.59-1.28)$ & & $0.39(0.24-0.61)$ & \\
\hline$>0.80$ & $0.72(0.41-1.28)$ & & $0.33(0.21-0.52)$ & \\
\hline
\end{tabular}

Model 1 includes the CD4+ and CD8+ T cell counts themselves, and Model 2 includes the CD4+:CD8+ T cell ratio. For each model, estimates on the left (A) reflect the estimates from a model including covariates at treatment initiation (baseline) only, whereas estimates on the right (B) are from models including the latest (time updated) measurements.

Adjusted for age, sex, ethnicity, mode of HIV acquisition, year of treatment initiation, HBV, HCV, initial treatment regimen and HIV viral load.

the UK CHIC cohort. ${ }^{11}$ Another reason for this difference maybe access to better treatments, in particular, INSTIs. In our study, $11 \%$ of those initiating treatment were prescribed an INSTI as a first-line drug, compared to only $2 \%$ in the Thai cohort, reflecting the earlier period of follow-up of the latter. A study from a French cohort reported that initiation of cART with an INSTI regimen was strongly associated with a faster rate of ratio normalization when compared to initiation with non-INSTI containing regimens. ${ }^{12}$

Despite the apparent slower ratio normalization in the Thai cohort, by 10 years of follow-up, almost $40 \%$ of the Thai cohort had experienced a normalized ratio, suggesting a cumulative beneficial effect of prolonged cART exposure and viral suppression. A study comparing Asian and Caucasian people with HIV (PWH) by Petoumenos et al. in 2017 confirmed that the difference in the rate of ratio normalization is not explained by a difference in ability to achieve normalization per se, but by the lower CD4+ and $\mathrm{CD} 8+\mathrm{T}$ cell counts at treatment initiation in Asian populations. ${ }^{13}$ In our study, those initiating treatment at lower CD4+ T cell counts or with lower CD4+:CD8+ T cell ratios still experienced improvements in their ratio over time. Importantly, the increase in $\mathrm{CD} 4+\mathrm{CD} 8+\mathrm{T}$ cell ratio is often believed to be due to the expansion of CD4+ T cells following viral suppression, with CD8+ T cells remaining abnormal. ${ }^{14}$ Further research into the imbalance of CD8+ T cell modulation in early HIV infection and persistence of elevated CD8+ $\mathrm{T}$ cells in later infection may help to understand why the CD4+:CD8+ $\mathrm{T}$ cell ratio does not restore to normal levels. ${ }^{15-17}$

We found multiple factors associated with ratio normalization, in addition to a higher CD4+ T cell count, lower CD8+ $\mathrm{T}$ cell count, and/or higher $\mathrm{CD} 4+\mathrm{CD} 8+\mathrm{T}$ cell ratio at treatment initiation. A diagnosis of an AIDS event before treatment initiation was associated with a reduced chance of ratio normalization, as also reported by others, ${ }^{18}$ likely reflecting the fact that individuals with clinical AIDS may have experienced additional immunological deficits, not captured through the $\mathrm{T}$ cell counts themselves, before treatment initiation. CD4+ T cell subsets are substantially affected in early infection. ${ }^{19-21}$ Although CD4+ T cell counts are observed to increase with initiation of cART and viral suppression, even at later stages of disease, the loss of balance in CD4+ T cell subsets during early infection may result in persistent immune activation and dysfunction. ${ }^{22,23}$

Those of black ethnicity were less likely to experience ratio normalization. This association has not previously been reported, but may reflect long-term immunological effects of late diagnoses and advanced disease, not adequately captured through the $\mathrm{T}$ cell counts. However, we did not account for continual treatment adherence after initial viral suppression in these analyses, and this may therefore be another explanation for these findings.

HBV infection before treatment initiation was associated with a lower chance of ratio normalization in multivariable models. This is similar to what has been reported previously from UK CHIC, ${ }^{5}$ although this association has not been seen in other cohort studies.

In line with other studies, ${ }^{23,24}$ we observed higher rates of ratio normalization with initiation of treatment using an INSTI in univariable analyses, although this association did not remain significant after adjustment. Our findings are likely to be limited by the relatively short follow-up time available in the era of treatment with INSTIs. Those receiving cART regimens, including TDF/FTC, were most likely to experience ratio normalization in 
comparison to those receiving regimens, including $\mathrm{ABC} / \mathrm{TTC}$ or ZDV/TTC.

Our study also found strong associations between both the most recent CD4+ $\mathrm{T}$ cell count and the CD4+:CD8+ $\mathrm{T}$ cell ratio and the development of a new AIDS event in both univariable and multivariable models. In contrast, we found no association between these measures when assessed at cART initiation (fixed covariate analysis), suggesting these cART-related changes to these values overwhelm any longerterm prognostic value of pre-cART values. These findings are similar to those reported by Mussini et al. ${ }^{7}$ and more recently by Han et al., ${ }^{9}$ thus highlighting the importance of clinically reviewing the CD4+ $\mathrm{T}$ cell count and CD4+:CD8+ $\mathrm{T}$ cell ratio as important clinical biomarkers. ${ }^{24}$

Some limitations of our study include a lack of detailed information on non-AIDS events, which meant that we were unable to assess the association between the CD4+:CD8+ T cell ratio and non-AIDS events. Reported findings on this association are conflicting. ${ }^{4,7,9,25-27}$ Furthermore, our analyses may be restricted by the lack of information on several unmeasured confounders in UK CHIC known to modulate immune responses, including infection by cytomegalovirus and other human herpesvirus, tobacco, and alcohol use. ${ }^{28-32}$ Finally, the small number of new AIDS events in the study means that our analyses may be underpowered to detect some associations.

\section{Conclusion}

This study provides an insight into the associations of CD4+ $\mathrm{T}$ cell count and $\mathrm{CD} 4+: \mathrm{CD} 8+\mathrm{T}$ cell ratio with ratio normalization and the development of a new AIDS event among PWH who achieved viral suppression on cART. As CD4+/CD8+ $\mathrm{T}$ cell counts and the CD4+:CD8+ T cell ratio were associated with ratio normalization, further research into the dynamics of T cell subsets, particularly CD8+ T cells, may help to understand why this is. It is important to continually assess CD4+ T cell count and CD4+:CD8+ T cell ratio due to their close relationship with the progression of HIV infection.

\section{Authors' Contributions}

H.O.: first author, design of work, analysis, interpretation, article writing, and corresponding author. M.J.V.G.: design of work, and writing and technical editing of article. T.H.: data collection and cleaning, and revision of article. C.A.S.: statistical analysis, writing and technical editing of article, and final overseeing of article submission. Authors agreed on all aspects of work for the final article.

\section{Steering Committee}

Jonathan Ainsworth, Sris Allan, Jane Anderson, Ade Apoola, David Chadwick, Duncan Churchill, Valerie Delpech, David Dunn, Ian Fairley, Ashini Fox, Richard Gilson, Mark Gompels, Phillip Hay, Rajesh Hembrom, Teresa Hill, Margaret Johnson, Sophie Jose, Stephen Kegg, Clifford Leen, Dushyant Mital, Mark Nelson, Hajra Okhai, Chloe Orkin, Adrian Palfreeman, Andrew Phillips, Deenan Pillay, Ashley Price, Frank Post, Jillian Pritchard, Caroline Sabin, Achim Schwenk, Anjum Tariq, Roy Trevelion, Andy Ustianowski, and John Walsh.

\section{Central Coordination}

University College London (David Dunn, Teresa Hill, Hajra Okhai, Andrew Phillips, and Caroline Sabin) and Medical Research Council Clinical Trials Unit at UCL (MRC CTU at UCL), London (Nadine van Looy and Keith Fairbrother).

\section{Participating Centers}

Barts Health NHS Trust, London (Chloe Orkin, Janet Lynch, and James Hand); Brighton and Sussex University Hospitals NHS Trust (Duncan Churchill, Stuart Tilbury, Elaney Youssef, and Duncan Churchill); Chelsea and Westminster Hospital NHS Foundation Trust, London (Mark Nelson, Richard Daly, David Asboe, and Sundhiya Mandalia); Homerton University Hospital NHS Trust, London (Jane Anderson and Sajid Munshi); King's College Hospital NHS Foundation Trust, London (Frank Post, Ade Adefisan, Chris Taylor, Zachary Gleisner, Fowzia Ibrahim, and Lucy Campbell); Middlesbrough, South Tees Hospitals NHS Foundation Trust, (David Chadwick and Kirsty Baillie); Mortimer Market Centre, University College London (Richard Gilson and Ian Williams); North Middlesex University Hospital NHS Trust, London (Jonathan Ainsworth, Achim Schwenk, Sheila Miller, and Chris Wood); Royal Free NHS Foundation Trust/University College London (Margaret Johnson, Mike Youle, Fiona Lampe, Colette Smith, Rob Tsintas, Clinton Chaloner, Caroline Sabin, Andrew Phillips, Teresa Hill, and Hajra Okhai); Imperial College Healthcare NHS Trust, London (John Walsh, Nicky Mackie, Alan Winston, Jonathan Weber, Farhan Ramzan, and Mark Carder); The Lothian University Hospitals NHS Trust, Edinburgh (Clifford Leen, Andrew Kerr, David Wilks, and Sheila Morris); North Bristol NHS Trust (Mark Gompels and Sue Allan); Leicester, University Hospitals of Leicester NHS Trust (Adrian Palfreeman and Adam Lewszuk); Woolwich, Lewisham and Greenwich NHS Trust (Stephen Kegg, Victoria Ogunbiyi, and Sue Mitchell), St. George's Healthcare NHS Trust (Phillip Hay, Christopher Hunt, Olanike Okolo, and Benjamin Watts); York Teaching Hospital NHS Foundation Trust (Ian Fairley, Sarah Russell-Sharpe, and Olatunde Fagbayimu); Coventry, University Hospitals Coventry and Warwickshire NHS Trust (Sris Allan and Debra Brain); Wolverhampton, The Royal Wolverhampton Hospitals NHS Trust (Anjum Tariq, Liz Radford, and Sarah Milgate); Chertsey, Ashford and St.Peter's Hospitals NHS Foundation Trust (Jillian Pritchard, Shirley Cumming, and Claire Atkinson); Milton Keynes Hospital NHS Foundation Trust (Dushyant Mital, Annie Rose, and Jeanette Smith); The Pennine Acute Hospitals NHS Trust (Andy Ustianowski, Cynthia Murphy, and Ilise Gunder); Nottingham University Hospitals NHS Trust (Ashini Fox, Howard Gees, Gemma Squires, and Laura Anderson), Kent Community Health NHS Foundation Trust (Rajesh Hembrom, Serena Mansfield, Lee Tomlinson, Christine LeHegerat, Roberta Box, Tom Hatton, and Doreen Herbert), The Newcastle upon Tyne Hospitals NHS Foundation Trust (Ashley Price, Ian McVittie, Victoria Murtha, and Laura Shewan); Derby Teaching Hospitals NHS Foundation Trust (Ade Apoola, Zak Connan, Luke Gregory, Kathleen Holding, Victoria Chester, Trusha Mistry, and Catherine Gatford); Public Health England, London (Valerie Delpech); and $i$-Base (Roy Trevelion). 


\section{Author Disclosure Statement}

C.A.S. has received funding for membership of Data Safety and Monitoring Boards and Advisory Boards, and for preparation of educational materials from Gilead Sciences and ViiV Healthcare. The other authors declare no conflicts of interest.

\section{Funding Information}

The UK CHIC Study is funded by the Medical Research Council, UK (grant nos. G0000199, G0600337, G0900274, and M004236).

\section{References}

1. Lu W, Mehraj V, Vyboh K, et al:: CD4:CD8 ratio as a frontier marker for clinical outcome, immune dysfunction and viral reservoir size in virologically suppressed HIV-positive patients. J Int AIDS Soc 2015;18: 20052.

2. Ananworanich J, Sacdalan CP, Pinyakorn S, et al.: Virological and immunological characteristics of HIV-infected individuals at the earliest stage of infection. J Virus Erad 2016;2:43-48.

3. Jenabian M-A, El-Far M, Vyboh $\mathrm{K}$, et al:: Immunosuppressive tryptophan catabolism and gut mucosal dysfunction following early HIV infection. J Infect Dis 2015;212:355-366.

4. Serrano-Villar S, Sainz T, Lee SA, et al: : HIV-infected individuals with low $\mathrm{CD} 4 / \mathrm{CD} 8$ ratio despite effective antiretroviral therapy exhibit altered $\mathrm{T}$ cell subsets, heightened CD8+ T cell activation, and increased risk of non-AIDS morbidity and mortality. PLoS Pathogens 2014; 10:e1004078.

5. Winston A, Jose S, Fisher M, et al.: Host, disease, and antiretroviral factors are associated with normalization of the CD4:CD8 ratio after initiating antiretroviral therapy. J Allergy Clin Immunol 2015;136:1682.e11685.e1.

6. The creation of a large UK-based multicentre cohort of HIV-infected individuals: The UK Collaborative HIV Cohort (UK CHIC) study. HIV Med 2004;5:115-124.

7. Mussini C, Lorenzini P, Cozzi-Lepri A, et al.: CD4/CD8 ratio normalisation and non-AIDS-related events in individuals with HIV who achieve viral load suppression with antiretroviral therapy: An observational cohort study. Lancet HIV 2015;2:e98-e106.

8. Tinago W, Coghlan E, Macken A, et al.: Clinical, immunological and treatment-related factors associated with normalised CD4+/CD8+ T-cell ratio: Effect of naive and memory T-cell subsets. PLoS One 2014;9: e97011.

9. Han WM, Apornpong T, Kerr SJ, et al.: CD4/CD8 ratio normalization rates and low ratio as prognostic marker for non-AIDS defining events among long-term virologically suppressed people living with HIV. AIDS Res Ther 2018; 15:13.

10. Strindhall J, Skog M, Ernerudh J, et al.: The inverted CD4/CD8 ratio and associated parameters in 66-year-old individuals: The Swedish HEXA immune study. AGE 2013;35:985-991.

11. Hoenigl M, Chaillon A, Little SJ: CD4/CD8 cell ratio in acute HIV infection and the impact of early antiretroviral therapy. Clin Infect Dis 2016;63:425-426.
12. De Salvador-Guillouet F, Sakarovitch C, Durant J, et al.: Antiretroviral regimens and CD4/CD8 ratio normalization in HIV-infected patients during the initial year of treatment: A cohort study. PLoS One 2015;10:e0140519.

13. Petoumenos K, Choi JY, Hoy J, et al.: CD4:CD8 ratio comparison between cohorts of HIV-positive Asians and Caucasians upon commencement of antiretroviral therapy. Antivir Ther 2017;22:659-668.

14. Helleberg M, Kronborg G, Ullum H, et al:: Course and clinical significance of CD8+ T-cell counts in a large cohort of HIV-infected individuals. J Infect Dis 2015;211:17261734.

15. Mudd JC, Lederman MM: CD8 T cell persistence in treated HIV infection. Curr Opin HIV AIDS 2014;9:500-505.

16. Ndumbi P, Gillis J, Raboud J, et al.: Characteristics and determinants of T-cell phenotype normalization in HIV-1infected individuals receiving long-term antiretroviral therapy. HIV Med 2014;15:153-164.

17. Cao W, Mehraj V, Kaufmann DE, Li T, Routy JP: Elevation and persistence of CD8 T-cells in HIV infection: The Achilles heel in the ART era. J Int AIDS Soc 2016;19: 20697.

18. Falster K, Petoumenos K, Chuah J, et al.: Poor baseline immune function predicts an incomplete immune response to combination antiretroviral treatment despite sustained viral suppression. JAIDS J Acq Imm Def Syndr 2009;50: 307-313.

19. Okoye AA, Picker LJ: CD4+T-cell depletion in HIV infection: Mechanisms of immunological failure. Immunol Rev 2013;254:54-64.

20. Lindqvist M, Van Lunzen J, Soghoian DZ, et al:: Expansion of HIV-specific $\mathrm{T}$ follicular helper cells in chronic HIV infection. J Clin Invest 2012;122:3271-3280.

21. Brenchley JM, Paiardini M, Knox KS, et al:: Differential Th17 CD4 T-cell depletion in pathogenic and nonpathogenic lentiviral infections. Blood 2008;112: 2826-2835.

22. Cecchinato V, Trindade CJ, Laurence A, et al.: Altered balance between Th17 and Th1 cells at mucosal sites predicts AIDS progression in simian immunodeficiency virus-infected macaques. Mucosal Immunol 2008;1:279288.

23. Favre D, Lederer S, Kanwar B, et al.: Critical loss of the balance between Th17 and T regulatory cell populations in pathogenic SIV infection. PLoS Pathogens 2009;5: e1000295.

24. Lee SS, Wong NS, Wong BCK, et al.: Combining CD4 recovery and $\mathrm{CD} 4$ : $\mathrm{CD} 8$ ratio restoration as an indicator for evaluating the outcome of continued antiretroviral therapy: An observational cohort study. BMJ Open 2017; 7:e016886.

25. Hema MN, Ferry T, Dupon M, et al.: Low CD4/CD8 ratio is associated with non AIDS-defining cancers in patients on antiretroviral therapy: ANRS CO8 (Aproco/ Copilote) prospective cohort study. PLoS One 2016;11: e0161594.

26. Castilho JL, Shepherd BE, Koethe J, et al.: CD4+/CD8+ ratio, age, and risk of serious noncommunicable diseases in HIV-infected adults on antiretroviral therapy. AIDS (London, England) 2016;30:899-908.

27. Menozzi M, Zona S, Santoro A, et al.: CD4/CD8 ratio is not predictive of multi-morbidity prevalence in HIVinfected patients but identify patients with higher CVD risk. J Int AIDS Soc 2014;17:19709. 
28. Gianella S, Moser C, Vitomirov A, et al:: Presence of asymptomatic cytomegalovirus and Epstein-Barr virus DNA in blood of persons with HIV starting antiretroviral therapy is associated with non-AIDS clinical events. AIDS 2020;34:849-857.

29. Ramendra R, Isnard S, Lin J, et al.: CMV seropositivity is associated with increased microbial translocation in people living with HIV and uninfected controls. Clin Infect Dis 2019;10:1093:ciz1001.

30. Morris SR, Chen B, Mudd JC, et al: Inflammescent CX3CR1+CD57+CD8+ T cells are generated and expanded by IL-15. JCI Insight 2020;5:132963.

31. Valiathan R, Miguez MJ, Patel B, Arheart KL, Asthana D: Tobacco smoking increases immune activation and impairs T-cell function in HIV infected patients on antiretrovirals: A cross-sectional pilot study. PLoS One 2014;9:e97698.
32. Monnig MA: Immune activation and neuroinflammation in alcohol use and HIV infection: evidence for shared mechanisms. Am J Drug Alcohol Abuse 2017;43:7-23.

Address correspondence to: Hajra Okhai Centre for Clinical Research, Epidemiology, Modelling and Evaluation Institute for Global Health University College London Royal Free Campus Rowland Hill Street London NW3 2PF United Kingdom

E-mail: h.okhai@ucl.ac.uk 